International Journal of Pure and Applied Mathematics

Volume 89 No. 5 2013, 743-760

ISSN: 1311-8080 (printed version); ISSN: 1314-3395 (on-line version)

url: http://www.ijpam.eu

doi: http://dx.doi.org/10.12732/ijpam.v89i5.10

ijpam.eu

\title{
REGIONAL QUADRATIC PROBLEM FOR DISTRIBUTED BILINEAR SYSTEMS WITH BOUNDED CONTROLS
}

\author{
Maawiya Ould Sidi ${ }^{\S}$, Sid Ahmed Ould Beinane ${ }^{2}$ \\ ${ }^{1,2}$ Departement of Mathematics \\ College of Sciences \\ Al Jouf University \\ Sakakah, KINGDOM OF SAUDI ARABIA
}

\begin{abstract}
In this paper, we establish the approximate controllability of a class of distributed bilinear systems evolving in a spatial domain $\Omega$. A bounded feedback control is used to drive a dynamical system from an initial state to a desired one in finite time, only on a subregion $\omega$ of the system domain. Our purpose is to prove that an regional optimal control exists, and characterized as a solution to an optimality system. Numerical approach is given and successfully illustrated by simulations.
\end{abstract}

AMS Subject Classification: 93B05, 93B52, 49K35

Key Words: distributed systems, bilinear systems, quadratic problems, regional bounded control, simulations

\section{Introduction}

Let $\Omega$ be an open bounded domain in $\mathbb{R}^{n}(n=1,2,3)$, with a regular boundary $\partial \Omega$. For $T>0$ we denote by $\Pi=\Omega \times] 0, T[, \Sigma=\partial \Omega \times] 0, T[$ and consider the infinite dimensional bilinear evolution system

Received: September 20, 2013

(C) 2013 Academic Publications, Ltd. url: www.acadpubl.eu

${ }^{\S}$ Correspondence author 


$$
\begin{cases}\frac{\partial u(x, t)}{\partial t}=A u(x, t)+Q(t) B u(x, t) & \Pi \\ u(x, 0)=u_{0}(x) & \Omega \\ u(x, t)=0 & \Sigma\end{cases}
$$

where $A$ generates a strongly continuous semigroup $(S(t))_{t \geq 0}$ of bounded linear operators the Banach space $L^{2}(\Omega)$ see [11]. We assume that $B: L^{2}(\Omega) \longrightarrow L^{2}(\Omega)$ is a linear bounded operator.

Let $U_{M}=\left\{Q \in L^{\infty}([0, T]) \mid-M \leq Q(t) \leq M\right\}$ the control space, $M$ is a positif constant. The system (1) is bilinear relative to the pair $(Q, u)$, and its solution $u$ is a nonlinear function on $u$ see [2].

For a given $u_{0} \in L^{2}(\Omega)$, (1) written as

$$
u_{Q}(x, t)=S(t) u_{0}(x)+\int_{0}^{t} S(t-s) Q(s) B u_{Q}(x, s) d s .
$$

and solutions of (2) are often called mild solutions of (1). The existence of a unique solution $u_{Q}(x, t)$ in $\mathcal{C}\left([0, T] ; L^{2}(\Omega)\right)$ satisfying (2), follows from standard results in [2].

The interest of these systems lies in the fact that many naturel and industrial process have intrinsically bilinear structure.

The increasing demand for groundwater as a drinking water resource requires the development of remediation methods. The damage caused by contaminants to the groundwater varies spatially due to the migration of the contaminants through the water and soil. Optimal control strategies for realistic contaminant transport models can expedite the remediation efforts.

Consider saturated flow through an idealized tube packed with soil which contains some contaminant. the initial concentration of contaminant along the length of the tube is known. A fluid will be pumped through the tube to remove the contaminant. The contaminant concentration is represented by $u(x, t)$ with $\mathrm{x}$ denoting the length along the tube axis measured from flow entrance $(x=0)$ to its total length $(x=L)$ and $\mathrm{t}$ denoting time. The contaminant concentration satisfies the solute transport equation,

$$
\begin{cases}\frac{\partial u(x, t)}{\partial t}=\kappa \frac{\partial^{2} u(x, t)}{\partial x^{2}}-v(t) \frac{\partial u(x, t)}{\partial x} & ] 0, L[\times] 0, T[ \\ u(x, 0)=u_{0}(x) & ] 0, L[ \\ u(0, t)=0 & ] 0, T[ \\ u_{x}(L, t)=0 & ] 0, T[\end{cases}
$$

The function $v(t)$ is the convective velocity due to the fluid pumped into the tube. The coefficient $\kappa$ is the "dispersion-diffusion" coefficient, which reflects 
the effects of the diffusion of the solute in the water as well as the dispersive effects of the water flowing through the soil pores.

To understand optimal control of the parabolic bilinear equation (3) governing groundwater flow, consider the performance criterion (cost functional)

$$
\min _{v \in U_{M}}\left[\alpha \int_{0}^{L} u(x, T) d x+\frac{\beta}{2} \int_{0}^{T} v^{2}(t) d t\right]
$$

for a positif constants $\alpha, \beta$ which is a combination of the final amount of contaminant and the cost of applying the control. Lenhart 1995 characterized the optimal control solution of (4).

For background on infinite dimensional distributed bilinear systems as (1), see [2]. To point at some of the relevant literature for problem under investigation $[9,3]$. They used controls acting through $Q(t) u$.

Here our control being a time-dependent scalar coefficient in the bilinear term $Q(t) B u$ as considered by [1], but for more general operator $A$ and $B$.

Recently the concept of regional analysis for infinite dimensional systems were developed by El Jai and Zerrik, see [6], and concerns the study of the classical analysis notions (controllability, observability, stability and so on) only on a subregion $\omega$ of the system evolution domain $\Omega$.

The system (1) is said to be approximately controllable in $\omega \subset \Omega$, if for all $u^{d} \in L^{2}(\omega)$ and $\varepsilon>0$, there exist a control $Q \in L^{2}([0, T])$ such that

$$
\left\|\chi_{\omega} u_{Q}(x, T)-u^{d}(x)\right\|_{L^{2}(\omega)} \leq \varepsilon
$$

where $u^{d}$ is a desired state in the space $L^{2}(\Omega)$ and $\chi_{\omega}: L^{2}(\Omega) \longrightarrow L^{2}(\omega)$ is the restriction operator to $\omega$.

The principal reasons for considering these regional notions is that, they are close to real applications, there exists systems which are controllable in some subregion $\omega \subset \Omega$ but not controllable in the whole domain $\Omega$, and controlling regionally a system is cheaper than controlling it in the whole domain, see [6]. In the last ten years there has being an increasing interest in the study of regional analysis for systems of parabolic and hyperbolic equations, see for instance [6, 15], and [13].

In this paper our main result is solving the regional bilinear controllability problem of distributed systems expressed by

$$
\min _{Q \in U_{M}} J(Q)
$$

where $J$ is the functional

$$
J(Q)=\left\|\chi_{\omega} u_{Q}(T)-u^{d}\right\|_{L^{2}(\omega)}^{2}+\frac{\eta}{2}\|Q\|_{L^{2}([0, T])}^{2}
$$


$\eta>0$ is a positive constant.

This problem was considered by (Zerrik and al. 2011 see [14]) and solved using unbounded control set, which is difficult from numerical point of view. Here we deal with the problem (5), where the control is bounded, this case is the most natural, and often encountered in real applications. To characterize the optimal control solution of the above problem, we propose an approach based on quadratic cost control problem see [3], which involves the minimization of the norm control and the final state error. This is the aim of this paper and is organized as follows

We will discuss in the second section, the quadratic cost control problem associated to (5). We show the existence of an optimal control by a minimizing sequence argument. And, we derive a characterization for optimal controls, using the solution of an optimality system, that consists of the equation (1) coupled with an adjoint equation. In Section 3, we give a numerical approach which leads to an algorithm, that we illustrate in Section 4 through numerical simulations.

\section{Regional Quadratic Control Problem}

The goal of this section is to justify that the minimum of problem 5 can be achieved, and to characterize an optimal control $Q^{*} \in U_{M}$

Theorem 1. There exists a pair $\left(\bar{u}, Q^{*}\right) \in \mathcal{C}\left([0, T] ; L^{2}(\Omega)\right) \times U_{M}$, such that $\bar{u}$ is the unique solution of

$$
\begin{cases}\frac{\partial u(x, t)}{\partial t}=A u(x, t)+Q^{*}(t) B u(x, t) & \Pi \\ u(x, 0)=u_{0}(x) & \Omega \\ u(x, t)=0 & \Sigma\end{cases}
$$

and $Q^{*}$ is a optimal control which minimizes the objective functional $J(Q)$ over $U_{M}$.

Proof. The solutions $u_{Q}(x, t)$ of the equation (1) are weak solutions in $W=$ $L^{2}\left(0, T ; L^{2}(\Omega)\right)$ see $[10,5]$.

Using (2), and the bound $C$ of the strongly continuous semigroup $(S(t))_{t \geq 0}$ in all finite interval of $[0, T]$ see [11], we have

$$
\left\|u_{Q}(t)\right\|_{W} \leq C\left\|u_{0}\right\|_{L^{2}(\Omega)}+C\|B\| \int_{0}^{t}|Q(s)|\left\|u_{Q}(s)\right\|_{W} d s
$$


Using Gronwall inequality, see [2], we obtain

$$
\left\|u_{Q}(t)\right\|_{W} \leq C_{1} \exp (C\|B\| M T)
$$

with $C_{1}=C\left\|u_{0}\right\|_{L^{2}(\Omega)}$.

The set $\left\{J(Q) \mid Q \in U_{M}\right\}$ is nonempty and bounded from below. Let the minimizing sequence $\left\{Q_{n}\right\}, n=1,2 \ldots$, be such that

$$
J^{*}=\lim _{n \rightarrow+\infty} J\left(Q_{n}\right)=\inf _{Q \in U_{M}} J(Q)
$$

$J\left(Q_{n}\right)$ is then bounded, it follows that $\left\|Q_{n}\right\|_{L^{2}([0, T])}$ is also bounded.

Let $u_{n}(x, t)=u_{Q_{n}}(x, t)$, we conclude from (8), that $\left\|u_{n}\right\|_{W}$ are uniformly bounded independently of $n$.

From the priori estimates we deduce that

$$
\left\|A u_{n}\right\|_{W} \leq M_{1},\left\|Q_{n} B u_{n}\right\|_{W} \leq M_{2} \text {, and }\left\|u_{n}^{\prime}\right\|_{W} \leq M_{3}
$$

where $M_{i},\{i=1,2,3\}$ are positive constants.

From these bounds, there exist subsequences with the following convergence properties:

$$
\begin{array}{lll}
Q_{n} \rightarrow Q^{*} & \text { weakly in } & L^{2}(0, T) \\
u_{n} \rightarrow \bar{u} & \text { weakly in } & W \\
A u_{n} \rightarrow \chi & \text { weakly in } & W \\
Q_{n} B\left(u_{n}\right) \rightarrow \Lambda & \text { weakly in } & W \\
u_{n}^{\prime} \rightarrow \Psi & \text { weakly in } & W
\end{array}
$$

classical argument, see [10], we verify that $\bar{u}(0)=u_{0}$, and we can pass to the limit in equation (1), associated with $\left(Q_{n}, u_{Q_{n}}\right)$ as $n \longrightarrow \infty$. Then $\bar{u}^{\prime}=\Psi$, $A \bar{u}=\chi$ and $Q^{*} B \bar{u}=\Lambda$. Consequently $\bar{u}=u\left(Q^{*}\right)$.

Now we verify that $Q^{*}$ is an optimal control.

Using the lower semi continuity of the norms, (applying Fatou's Lemma), we deduce

$$
\begin{aligned}
J\left(Q^{*}\right) & =\inf _{n} \int_{\omega}\left(\chi_{\omega} u_{n}(x, T)-u_{d}\right)^{2}+\frac{\eta}{2} \int_{0}^{T} Q_{n}^{2}(t) d t \\
& \leq \lim _{n \longrightarrow \infty} J\left(Q_{n}\right)=\inf _{Q} J(Q)
\end{aligned}
$$

then $Q^{*}$ is an optimal control.

Remark 2. If we consider the state equation with a source term $f \in$ $L^{\infty}\left(0, T ; L^{2}(\Omega)\right)$,

$$
\frac{\partial u}{\partial t}=A u+Q(t) B u+f \quad \Pi
$$


the same well-posedness and regularity results as (1) hold, but the constant $C_{1}$ in (8), takes the form

$$
C_{1}=C\left(\left\|u_{0}\right\|_{L^{2}(\Omega)}+\|f\|_{L^{\infty}\left(0, T ; L^{2}(\Omega)\right)}\right) .
$$

To obtain a characterization of the optimal control, we must derive the optimality system by differentiating the cost functional $J(Q)$ with respect to the control $Q$.

We examine the differentiability of $Q \longrightarrow u(Q)$ with respect to $\mathrm{Q}$.

Lemma 3. The map

$$
Q \in U_{M} \longrightarrow u=u(Q) \in W
$$

is differentiable in the following sense:

$$
\frac{u(Q+\epsilon h)-u(Q)}{\epsilon} \rightarrow \psi \text { weakly in } W
$$

as $\epsilon \longrightarrow 0$ where $Q+\epsilon h \in U_{M}, h \in L^{\infty}([0, T])$. Moreover $\psi=\psi(u, h)$ satisfies

$$
\begin{cases}\psi_{t}(x, t)=A \psi(x, t)+Q(t) B \psi(x, t)+h(t) B \bar{u}(x, t) & \Pi \\ \psi(x, 0)=\psi_{0}(x)=0 & \Omega \\ \psi(x, t)=0 & \Sigma\end{cases}
$$

where $\bar{u}=u\left(Q^{*}\right)$.

Proof. Consider $u^{\epsilon}=u(Q+\epsilon h)$, then $\varphi(x, t)=\frac{u^{\epsilon}-u}{\epsilon}(x, t)$ is the weak solution of

$$
\begin{cases}\frac{\partial \varphi(x, t)}{\partial t}=A \varphi(x, t)+Q(t) B \varphi(x, t)+h(t) B u^{\epsilon} & \Pi \\ \varphi(x, 0)=0 & \Omega \\ \varphi(x, t)=0 & \Sigma\end{cases}
$$

Using (8) and Remark 2, we obtain

$$
\begin{aligned}
\left\|\frac{u^{\epsilon}-u}{\epsilon}\right\|_{W} & \leq\left\|h B u^{\epsilon}\right\|_{L^{\infty}\left(0, T ; L^{2}(\Omega)\right)} \exp (C M T\|B\|) \\
& \leq C_{2}
\end{aligned}
$$

where $C_{2}$ is independent of $\epsilon$ since the bound on $\left\|u^{\epsilon}\right\|_{L^{\infty}\left(0, T ; L^{2}(\Omega)\right)}$ is independent of $\epsilon$, and the weak convergence to $\psi$ is obtained. We also have

$$
\frac{\left(u^{\epsilon}-u\right)}{\epsilon} \longrightarrow \text { strongly in } L^{2}(\Omega) \text { as } \varepsilon \longrightarrow 0
$$


see [9], we conclude that $\psi$ satisfies (11).

We have also the weak convergence of the traces $\frac{\left(u^{\epsilon}-u\right)}{\epsilon}(x, T)$, in $L^{2}(\Omega)$ see [9].

We are ready to characterize the optimal control, by deriving the optimality system through differentiating $J(Q)$ with respect to $Q$ at an optimal control.

Theorem 4. Given an optimal control $Q^{*}$ in $U_{M}$, and the corresponding solution $\bar{u}=u\left(Q^{*}\right)$ to (1), the adjoint equation

$$
\begin{cases}\frac{\partial p(x, t)}{\partial t}=-A^{*} p(x, t)-Q^{*}(t) B^{*} p(x, t) & \Pi \\ p(x, T)=\left(\bar{u}(T)-\chi_{\omega}^{*} u^{d}\right) & \Omega \\ p(x, t)=0 & \Sigma\end{cases}
$$

has a unique solution $p \in W$.

$A^{*}$ the adjoint operator of the operator $A$, generates a strongly continuous semigroup $\left(S^{*}(t)\right)_{t \geq 0}$ of bounded linear operators on $L^{2}(\Omega)$, and $B^{*}$ is the linear bounded operator adjoint of the operator $B, \chi_{\omega}^{*}$ is the adjoint operator of $\chi_{\omega}$, defined from $L^{2}(\omega) \longrightarrow L^{2}(\Omega)$, and given by

$$
\chi_{\omega}^{*} u(x)=\left\{\begin{array}{cc}
u(x) & x \in \omega \\
0 & x \in \Omega \backslash \omega
\end{array}\right.
$$

Moreover,

$$
Q^{*}(t)=\max \left(-M, \min \left(-\frac{2}{\eta} \int_{\omega} \chi_{\omega} B \bar{u} \chi_{\omega} d x, M\right)\right)
$$

Proof. Consider $Q \in U_{M}$ and let $h \in L^{\infty}(0, T)$ such that $Q+\epsilon h \in U_{M}$ for 
$\epsilon>0$. The derivative of $J(Q)$ with respect to $Q$ in the direction $h$ satisfies.

$$
\begin{aligned}
& 0 \leq \lim _{\epsilon \longrightarrow 0} \frac{J(Q+\epsilon h)-J(Q)}{\epsilon} \\
& =\lim _{\epsilon \longrightarrow 0} \int_{\omega} \frac{\left(\chi_{\omega} u^{\epsilon}-u^{d}\right)^{2}-\left(\chi_{\omega} u-u^{d}\right)^{2}}{\epsilon} d x \\
& +\lim _{\epsilon \longrightarrow 0} \frac{\eta}{2} \int_{0}^{T} \frac{(Q+\epsilon h)^{2}-Q^{2}}{\epsilon}(t) d t \\
& =\lim _{\epsilon \longrightarrow 0} \int_{\omega} \chi_{\omega} \frac{\left(u^{\epsilon}-u\right)}{\epsilon}\left(\chi_{\omega} u^{\epsilon}+\chi_{\omega} u-2 u^{d}\right) d x \\
& +\lim _{\epsilon \longrightarrow 0} \frac{\eta}{2} \int_{0}^{T}\left(2 h Q+\epsilon h^{2}\right) d t \\
& =2 \int_{\omega} \chi_{\omega} \psi(x, T) \chi_{\omega}\left(u(x, T)-\chi_{\omega}^{*} u^{d}\right) d x \\
& +\eta \int_{0}^{T} h u d t \\
& =2 \int_{\omega} \chi_{\omega} \psi(x, T) \chi_{\omega} p(x, T) d x+\eta \int_{0}^{T} h Q d t \\
& =2 \int_{\omega} \chi_{\omega}^{*} \chi_{\omega}\left[\int_{0}^{T} \frac{\partial p}{\partial t} \psi d t+\int_{0}^{T} p \frac{\partial \psi}{\partial t} d t\right] d x \\
& +\eta \int_{0}^{T} h Q d t
\end{aligned}
$$

Integrating by parts, yields

$$
\begin{aligned}
0 & \leq \lim _{\epsilon \longrightarrow 0} \frac{J(Q+\epsilon h)-J(Q)}{\epsilon} \\
& =2 \int_{\omega} \chi_{\omega} \chi_{\omega}\left[\int_{0}^{T} \psi \frac{\partial p}{\partial t} d t+\int_{0}^{T} \frac{\partial \psi}{\partial t} p d t\right] d x \\
& +\eta \int_{0}^{T} h Q d t
\end{aligned}
$$

The system (11) gives

$$
\begin{aligned}
0 & \leq \frac{1}{2} \lim _{\epsilon \longrightarrow 0} \frac{J(Q+\epsilon h)-J(Q)}{\epsilon} \\
& =\int_{\omega} \chi_{\omega}^{*} \chi_{\omega} \int_{0}^{T} \psi \frac{\partial p}{\partial t} d t d x+\eta \int_{0}^{T} h Q d t \\
& +\int_{\omega} \chi_{\omega}^{*} \chi_{\omega}\left[\int_{0}^{T}(A \psi+Q(t) B \psi+h(t) B \bar{u}) p d t\right] d x
\end{aligned}
$$


And using the system (13) we obtain

$$
\begin{aligned}
0 & \leq \lim _{\epsilon \rightarrow 0} \frac{J\left(Q^{*}(t)+\epsilon h\right)-J\left(Q^{*}(t)\right)}{\epsilon} \\
& \leq 2 \int_{\omega} \chi_{\omega}^{*} \chi_{\omega}\left[\int_{0}^{T} \psi\left(\frac{\partial p}{\partial t}+A^{*} p+Q^{*}(t) B^{*} p\right) d t\right] d x \\
& +2 \int_{\omega} \chi_{\omega}^{*} \chi_{\omega} \int_{0}^{T} h(t) B \bar{u} p d t d x+\eta \int_{0}^{T} h Q^{*}(t) d t \\
& =2 \int_{0}^{T} h(t)\left\langle\chi_{\omega} B \bar{u} ; \chi_{\omega} p\right\rangle_{L^{2}(\omega)}+\eta \int_{0}^{T} h(t) Q^{*}(t) d t \\
& =\int_{0}^{T}\left[2\left\langle\chi_{\omega} B \bar{u}(x, t) ; \chi_{\omega} p(t)\right\rangle_{L^{2}(\omega)}+\eta Q^{*}(t)\right] h(t) d t
\end{aligned}
$$

Thus, using the arbitrary variation of $h$, and bounds on the control set, we have

$$
Q^{*}(t)=\max \left(-M, \min \left(-\frac{2}{\eta}\left\langle\chi_{\omega} B \bar{u} ; \chi_{\omega} p\right\rangle_{L^{2}(\omega)}, M\right)\right)
$$

\section{Numerical Approach}

Consider the one dimensional system

$$
\begin{cases}\left.\frac{\partial u(x, t)}{\partial t}=\lambda \frac{\partial^{2} u(x, t)}{\partial x^{2}}-Q(t) \beta u(x, t)\right] & ] 0,1[\times] 0, T[ \\ u(0)=u_{0} & ] 0,1[ \\ u(0, t)=u(1, t)=0 & ] 0, T[\end{cases}
$$

and the problem (5), which solution $Q^{*}$ is given by the formula

$$
Q^{*}(t)=\max \left(-M, \min \left(\frac{2 \beta}{\eta}\left\langle\chi_{\omega} \bar{u} ; \chi_{\omega} p\right\rangle_{L^{2}(\omega)}, M\right)\right)
$$

where $\bar{u}=u\left(Q^{*}\right)$ solution of $(20), p$ is solution of the adjoint equation associated to $(20)$, and given by

$$
\begin{cases}\left.-\frac{\partial p(x, t)}{\partial t}=\lambda \frac{\partial^{2} p(x, t)}{\partial x^{2}}-Q^{*}(t) \beta p(x, t)\right] & 0,1[\times] 0, T[ \\ p(T)=\left(\bar{u}(T)-\chi_{\omega}^{*} u^{d}\right), & ] 0,1[ \\ p(0, t)=p(1, t)=0 & ] 0, T[\end{cases}
$$


The following result enables us to simplify the above expression of $Q^{*}(t)$.

Proposition 5. Consider the system (20), for $M$ large enough, the optimal control is given by

$$
Q^{*}(t)=\frac{2 \beta}{\eta}\left\langle\chi_{\omega} \bar{u}(x, t) ; \chi_{\omega} p(x, t)\right\rangle_{L^{2}(\omega)}
$$

Proof. Since $Q=0 \in U_{M}$, we have,

$$
\begin{aligned}
J(0)=\left\|\chi_{\omega} u^{0}(T)-u^{d}\right\|_{L^{2}(\omega)}^{2} & \geq J\left(Q^{*}\right) \\
& \geq \frac{\eta}{2}\left\|Q^{*}(t)\right\|_{L^{2}(0, T)}^{2}
\end{aligned}
$$

where $u^{0}$ satisfies (20) with control $Q=0$ and $Q^{*} \in U_{M}$ is an optimal control. Thus $J(0)$ gives a bound of $\left\|Q^{*}(t)\right\|_{L^{2}(0, T)}^{2}$ that is independent of $\mathrm{M}$.

On an interval $(0, \tau)$ with $\tau \in[0, T], u=u\left(Q^{*}\right)$ the solution of (20) satisfy

$$
\begin{aligned}
\int_{0}^{1} \int_{0}^{\tau} \frac{\partial u(x, t)}{\partial t} u(x, t) d t d x & =\lambda \int_{0}^{1} \int_{0}^{\tau} \frac{\partial^{2} u}{\partial x^{2}} u d t d x \\
& -Q^{*}(t) \beta u^{2}(x, t) d t d x
\end{aligned}
$$

So

$$
\begin{aligned}
\int_{0}^{1}\left[\frac{u^{2}(x, t)}{2}\right]_{0}^{\tau} d x & =-\lambda \int_{0}^{\tau} \int_{0}^{1}\left(\frac{\partial u(x, t)}{\partial x}\right)^{2} d x d t \\
& -\int_{0}^{1} \int_{0}^{\tau} Q^{*}(t) \beta u^{2}(x, t) d t d x
\end{aligned}
$$

which gives

$$
\begin{aligned}
\frac{1}{2} \int_{0}^{1} u^{2}(x, \tau) d x & =\frac{1}{2} \int_{0}^{1} u_{0}^{2}(x) d x \\
& +\frac{\beta}{2} \int_{0}^{1} \int_{0}^{\tau}\left(Q^{*}\right)^{2}(t) u^{2}(x, t) d t d x \\
& +\frac{\beta}{2} \int_{0}^{1} \int_{0}^{\tau} u^{2}(x, t) d t d x
\end{aligned}
$$


Rearranging yields

$$
\begin{aligned}
\int_{0}^{1} u^{2}(x, \tau) d x & =\int_{0}^{1} u_{0}^{2}(x) d x \\
& \left.+\int_{0}^{\tau} \frac{\beta}{2}\left(Q^{*}\right)^{2}(t)+1\right)\left(\int_{0}^{1} u^{2} d x\right) d t
\end{aligned}
$$

and Gronwall's inequality implies,

$$
\begin{aligned}
\int_{0}^{1} u^{2}\left(x, Q^{*}\right) d x & \leq\left\|u_{0}\right\|_{L^{2}([0,1])}^{2} \exp \frac{\beta}{2}\left(\left\|Q^{*}(t)\right\|_{L^{2}(0, T)}^{2}+T\right) \\
& \leq\left\|u_{0}\right\|_{L^{2}([0,1])}^{2} \exp \left(\frac{\beta}{2} J(0)+\frac{\beta T}{2}\right)
\end{aligned}
$$

which is bounded independently of $M$.

And by the same method, one obtain

$$
\begin{aligned}
\int_{0}^{1} p^{2}\left(x, Q_{\eta}\right) d x & \leq\|p(T)\|_{L^{2}([0,1])}^{2} \exp \frac{\beta}{2}\left(\left\|Q^{*}(t)\right\|_{L^{2}(0, T)}^{2}+T\right) \\
& \leq\|p(T)\|_{L^{2}([0,1])}^{2} \exp \left(\frac{\beta}{2} J(0)+\frac{\beta T}{2}\right)
\end{aligned}
$$

which is also bounded independently of $M$.

From (19) and for $\omega \in] 0,1[$, we obtain

$$
\begin{aligned}
Q^{*}(t) & \leq\left|Q^{*}(t)\right| \\
& \leq \frac{2 \beta}{\eta}\left(\int_{0}^{1} u^{2} d x\right)^{1 / 2}\left(\int_{0}^{1} p^{2} d x\right)^{1 / 2} \\
& \leq M_{1}
\end{aligned}
$$

where $M_{1}$ is independent of $M$.

For $M>M_{1},(21)$ becomes

$$
Q^{*}(t)=\frac{2 \beta}{\eta}\left\langle\chi_{\omega} \bar{u}(x, t) ; \chi_{\omega} p(t)\right\rangle_{L^{2}(\omega)} .
$$


Corollary 6. An optimal control $Q^{*}$, the corresponding state $\bar{u}$, and the adjoint state $p$ are necessarily linked by the following relations:

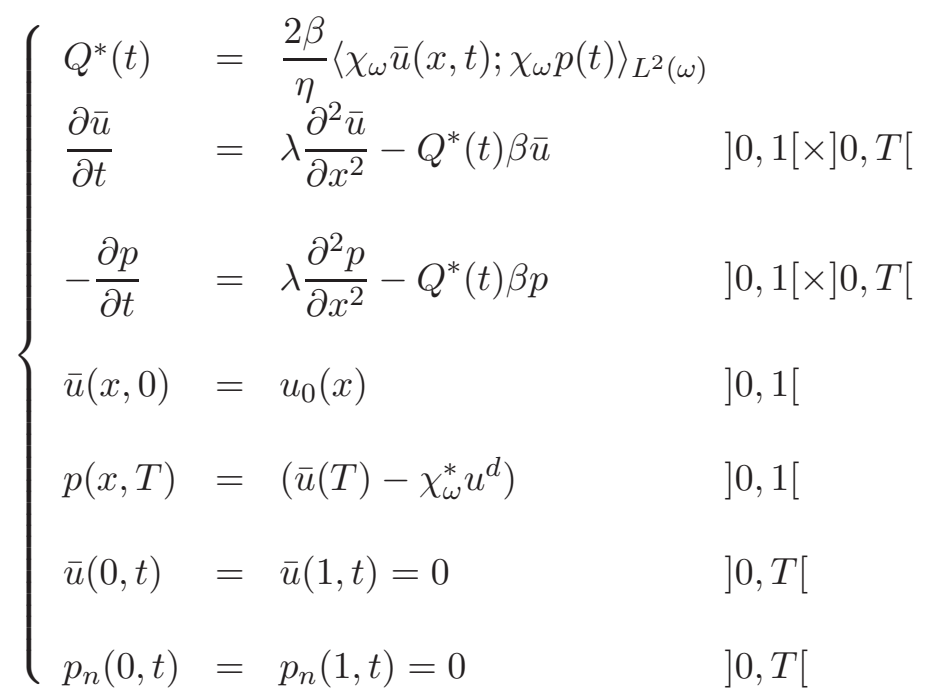

The computation of an optimal control, solution of the problem (5), can be realized by the following formula

$$
\left\{\begin{aligned}
Q_{n+1}(t) & =\frac{2 \beta}{\eta}\left\langle\chi_{\omega} u_{n}(x, t) ; \chi_{\omega} p_{n}(t)\right\rangle_{L^{2}(\omega)} \\
Q_{1} & =0
\end{aligned}\right.
$$

where $u_{n}$ is the solution of (20) associated with $Q_{n}$, and $p_{n}$ is the solution of the adjoint equation

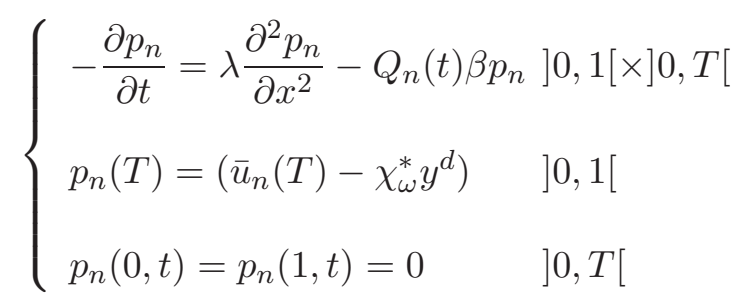

which allows to consider the following algorithm

\section{Algorithm}




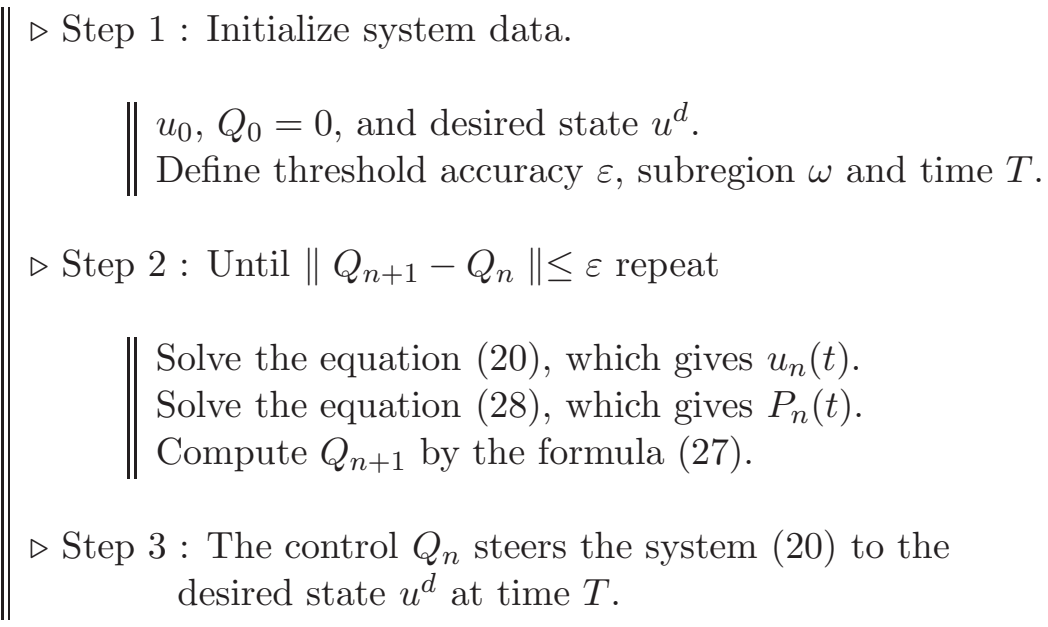

\section{Simulations}

Consider the system (20), with $\lambda=\beta=0.01$, and the problem (5) with $\eta=\frac{1}{5}$. We take $u_{0}(x)=2 x\left(1-x^{3}\right)$, and the desired state $u^{d}(x)=0.94 x(x-1)(x+1)$. Applying the previous algorithm for different subregions, we obtain:

- Case of $\omega=] 0.25,0.55[$, and $T=1$,

Figure 1 shows how the reached state (solid line) is very close to the desired position (dotted line) on $\omega$. The desired state is obtained with error $\mathcal{E}=\left\|\bar{u}(T)-u^{d}\right\|_{L^{2}(\omega)}^{2}=3.09 \times 10^{-4}$ and cost $\left\|Q_{4}^{*}\right\|^{2}=1.53 \times 10^{-5}$.

- Case of $\omega=] 0,0.2[$, and $T=2$,

Figure 3 shows how the reached state (solid line) is very close to the desired one (dotted line) on $\omega$. The desired state is obtained with error $\mathcal{E}=\left\|\bar{u}(T)-u^{d}\right\|_{L^{2}(\omega)}^{2}=6.01 \times 10^{-4}$, and cost $\left\|Q_{4}^{*}\right\|^{2}=4.1 \times 10^{-6}$.

- Case of $\omega=\Omega=] 0,1[$, and $T=2$,

Figure 5 shows how the reached state (solid line) is very close to the desired one (dotted line) on $\Omega$. The desired state is obtained with error $\mathcal{E}=\left\|\bar{u}(T)-u^{d}\right\|_{L^{2}(\omega)}^{2}=1.8 \times 10^{-2}$, and cost $\left\|Q_{4}^{*}\right\|^{2}=1.47 \times 10^{-4}$. 


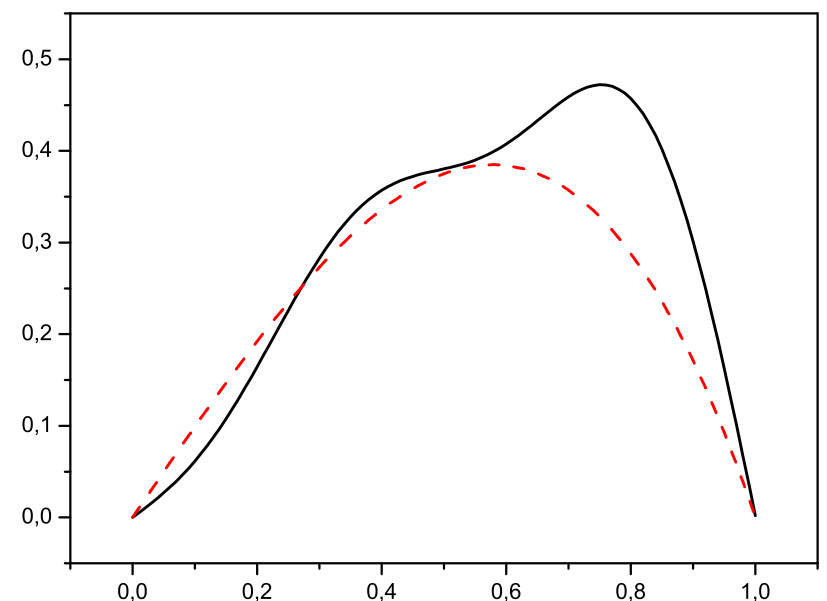

Figure 1: Desired (...) and final state $(-)$ on $\omega$.

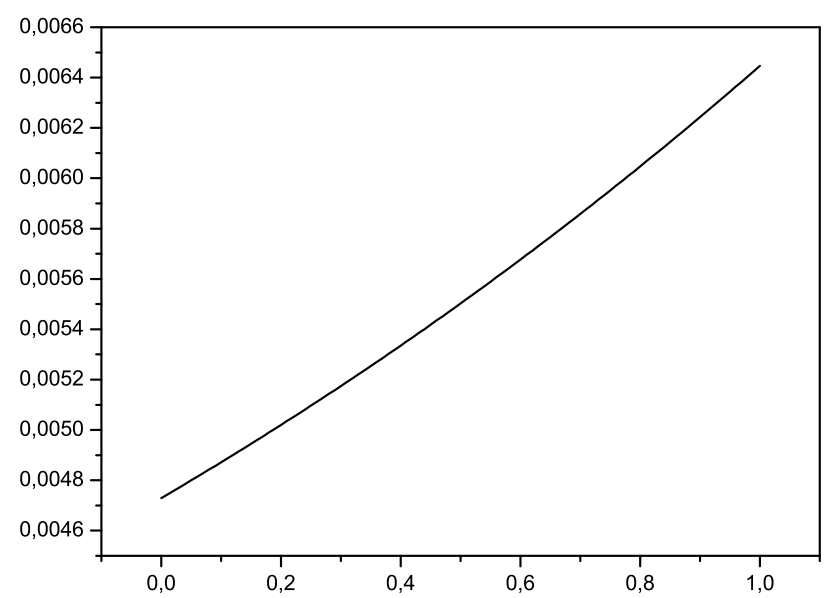

Figure 2: Evolution of the control function $Q_{4}^{*}$ on $[0,1]$.

\section{Conclusion}

We consider a regional quadratic problem for distributed bilinear systems. The developed approach allows us to asses the fitness of the finale state to a pre- 


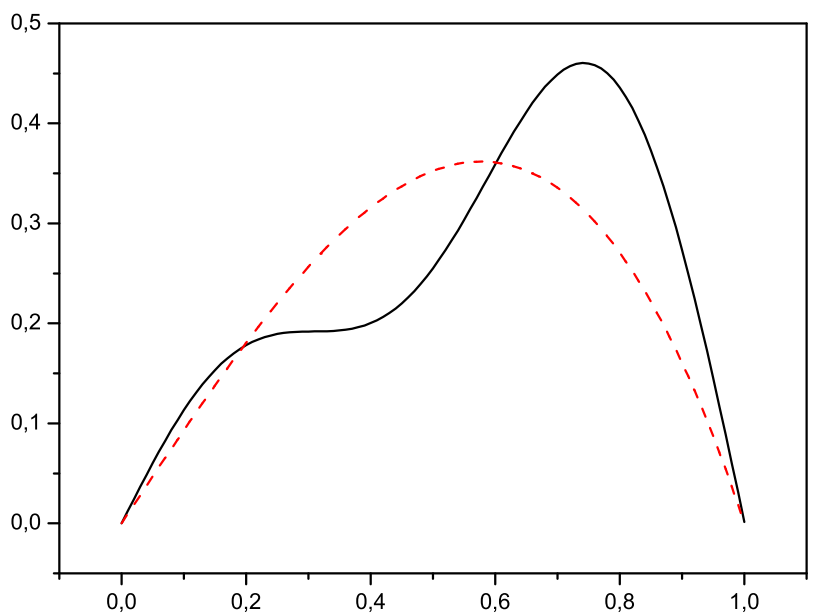

Figure 3: Desired (...) and final state $(-)$ on $\omega$.

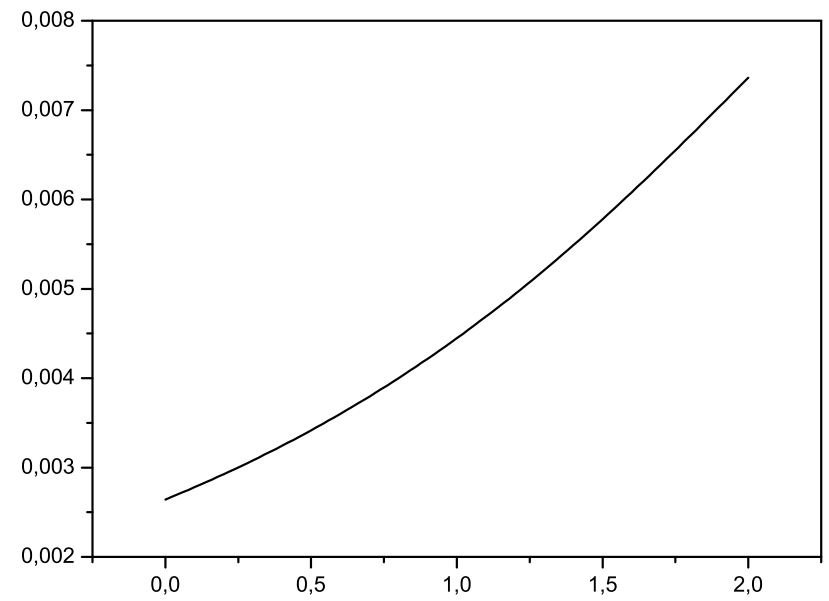

Figure 4: Evolution of the control function $Q_{4}^{*}$ on $[0,2]$.

scribed target restricted to some subdomain of the system space domain.

This is achieved under a bounded set of control. The existence of an opti- 


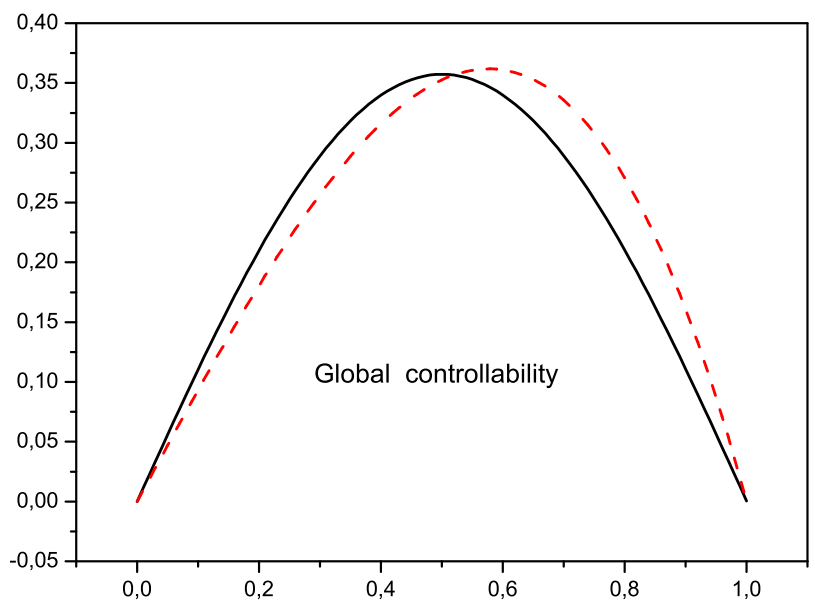

Figure 5: Desired (...) and final state $(-)$ on $\Omega$.

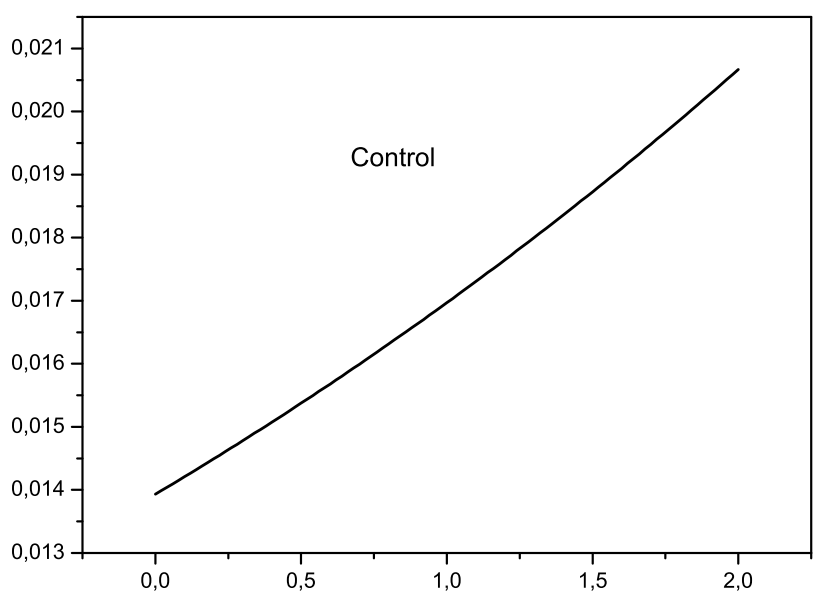

Figure 6: Evolution of the control function $Q_{4}^{*}$ on $[0,2]$.

mal control is proved and characterized through the differentiability method. Moreover, we developed a numerical approach that leads to implicit formula for the optimal control for the regional quadratic control problem. The obtained 
results are successfully tested through numerical examples. Questions are still open: the case of bilinear hyperbolic system, which is under consideration, and the work will appear in a separate paper.

\section{References}

[1] A. Addou, A. Benbrik, . Existence and uniqueness of optimal control for a distributed-parameter bilinear system, Journal of dynamical and control systems, 8 (2002), 141-152.

[2] J. Ball, J.E. Marsden, and M. Slemrod, . Controllability for Distributed Bilinear systems, SIAM J. on control and opt, 40 (1982), 575-597.

[3] M. E. Bradly, S. Lenhart, Bilinear optimal control of a Kirchhoff plate to a desired profile, Journal of Optimal control applications \& methods, 18 (1997), 217-226.

[4] M. E. Bradly, S. Lenhart, and J. Yong, . Bilinear Optimal control of the Velocity term in a Kirchhoff plate equation, Journal of Mathematical Analysis and Applications, 238 (1999), 451-467.

[5] H. Brezis, Analyse fonctionnelle : théorie et application, Masson, (1983).

[6] A. El Jai, A.J. Pritchard, M. C. Simon, and E. Zerrik, Regional controllability of distributed systems, International Journal of Control, 62 (1995), 1351-1365.

[7] A. Kamal, A. Boutoulout, And S. A. Ould Beinane, Regional boundary controllability of semi-linear distributed parabolic system, International Review of Automatic Control, 3 (2010), 378-387.

[8] A. Kamal, A. Boutoulout And S. A. Ould Beinane, Regional Controllability of Semi-Linear Distributed Parabolic Systems: Theory and Simulation, Intelligent Control and Automation,3 (2012), 146-158.

[9] S. Lenhart, Optimal control of a convective-diffusive fluid problem, Math. Models Method Appl. Sci., 5 (1995), 225-237.

[10] J.L. Lions, E. Magenes, Problèmes aux limites non homogènes et applications, Vol. 1 et 2. Dunod, (1968).

[11] A. Pazy, Semigroups of linear operators and applications to partial differential equations, Springer-Verlag, New-York, (1983). 
[12] E. Zerrik, M. Ould Sidi, An output controllability of bilinear distributed system, International Review of Automatic Control, 3 (2010), 466-473.

[13] E. Zerrik, M. Ould Sidi, Regional controllability of linear and semi linear hyperbolic systems, Int. Journal of Math. Analysis, 4 (2010), 2167-2198.

[14] E. Zerrik, M. Ould Sidi, Regional Controllability for Infinite Dimensional Distributed Bilinear Systems, Int. Journal of control, 84 (2011 ), 21082116.

[15] E. Zerrik, M. Ould Sidi, Constrained regional control problem for distributed bilinear systems, IET Cont. Theory. Appl., 7 (2013), 1914-1921. 